\title{
Galectin-9 and PSMB8 overexpression predict unfavorable prognosis in patients with $A M L$
}

\author{
Yongping Zhang ${ }^{*}$, Song Xue*, Qi Hao, Fuhong Liu, Wenqiu Huang, Jingbo Wang ${ }^{\bowtie}$ \\ Department of Hematology, Aerospace Center Hospital, Peking University Aerospace School of Clinical Medicine, Beijing 100049, China. \\ *These authors contributed equally to this work. \\ $\bowtie$ Corresponding author: E-mail: wangjingbo@asch.net.cn.
}

(c) The author(s). This is an open access article distributed under the terms of the Creative Commons Attribution License (https://creativecommons.org/licenses/by/4.0/). See http:/ /ivyspring.com/terms for full terms and conditions.

Received: 2020.09.24; Accepted: 2021.05.10; Published: 2021.05.19

\begin{abstract}
Acute myeloid leukemia (AML) is a deadly heterogeneous hematologic malignancy. Despite the well-characterized genetic characteristics and new promising targeted therapies for AML, the clinical outcome remains suboptimal. Galectin-9 (Gal-9) is a good potential target due to its immunosuppressive capacity in inflammatory processes. In our study, we firstly performed a wide range of integrated bioinformatical approach to assess the importance of $\mathrm{Gal}-9$ by analyzing the expression, potential function and prognostic impact in AML. The results indicated that Gal-9 is overexpressed in AML cells, especially when relapse after hematopoietic stem cell transplantation (HSCT) and predicts poor prognosis. Co-expression analysis showed Gal-9 has a strong positive correlation with proteasome subunit beta type-8 (PSMB8), which was also highly expressed in AML with poor prognosis, implying a synergy in cell survival, cell signaling and the development of AML. In summary, we have confirmed the overexpression of Gal-9 and its partner PSMB8 in AML and validated their importance as prognostic factors. We propose that Gal-9 and PSMB8 could be a promising molecular target for treatment of AML and may provide more combined treatment options, especially in patients with relapse after HSCT.
\end{abstract}

Key words: acute myeloid leukemia (AML); Galectin-9 (Gal-9); bioinformatics analysis; proteasome subunit beta type-8 (PSMB8); biomarker; prognosis

\section{Introduction}

AML is a clinically and genetically heterogeneous disease that originates from hematopoietic stem and progenitor cells (HSPCs) characterized by differentiation blockade and clonal proliferation of immature blast cells reside in bone marrow, peripheral blood and extramedullary sites suppressing normal hematopoiesis[1]. It is the most common acute leukemia in adults with an incidence that increases with age [2]. The standard of induction chemotherapy regimen consisting of cytarabine and anthracycline for AML was established over the last decades and remains profoundly unchanged today [1]. Even though up to $85 \%$ younger patients $(\leq 60$ years) patients respond to induction chemotherapy and achieve complete remission, the most patients will eventually relapse within 3 years [1]. Only about $25 \%$ AML patients survives 5 years or longer in spite of multidrug combined chemotherapy, molecular targeted therapies and HSCT [3]. Prognosis is even worse in elderly patients (>60 years) and those who cannot tolerate standard induction chemotherapy, with a median survival of only 5-10 months and 5 -year overall survival (OS) of $5 \%[1,4,5]$. Thus, there is an urgent need for the development of novel therapeutic approaches for AML.

One promising molecular target is the Gal-9, a member of the ninth member of the $\beta$-galactoside-binding soluble lectin family consists of two carbohydrate recognition domains linked by a peptide, and it was first identified as an eosinophil chemoattractant and activation factor [6, 7]. The Gal-9 gene LGALS9 is localized in the short arm of human chromosome 17, which has several isoforms [8]. Gal-9 is found in both the cytoplasm and extracellular regions [9] and expressed on immune cells, parenchymal cells and a variety types of tumor cells 
[10]. Gal-9 has been shown to regulate many different biological functions such as inflammation, cell adhesion, cell proliferation, and cell apoptosis $[8,10$, 11]. Numerous studies have shown that the interactions between Gal-9 and its ligand, $\mathrm{T}$ cell immunoglobulin mucin-3 (Tim-3), negatively regulates $\mathrm{T}$ cell responses through promoting Th1, Th17 and cytotoxic T cells exhaustion, driving the expansion of myeloid-derived suppressor cells and FoxP3+T regulatory cells (Tregs) [11, 12]. As Gal-9 was highly expressed on AML cells [10], which may result in tumorigenicity, tumor growth and escape from immunity and Gal-9 may be a biomarker of poor prognosis for AML. In this work, we firstly performed a wide range of integrated bioinformatical approach to assess the importance of Gal-9 by analyzing the expression, potential function and prognostic impact of Gal-9 in human AML.

\section{Methods}

\section{Bioinformatics Mining for Identifying Gal-9 Expression}

GEPIA

(http://gepia2.cancer-pku.cn) and Oncomine (https://www.oncomine.org/resource/main. $h \mathrm{tml}$ ) cancer databases were mined to determine the Gal-9 differential expression level between an AML group and a normal group. GEPIA is a newly developed interactive web server for analyzing the RNA sequencing expression data of 9736 tumors and 8587 normal samples from the TCGA and the GTEx projects, using a standard processing pipeline [13]. The Oncomine database is a publicly accessible, online cancer microarray database that helps facilitate research from genome-wide expression analysis. The fold change was defined as $>2$ and the gene ranks in the top $10 \%$, p value was set up at $<1 \mathrm{E}-4$. The mRNA levels of Gal-9 in a series of cancers were analyzed by CCLE database (https://portals.broadinstitute.org/ccle/ about), which is an online encyclopedia of a compilation of gene expression, chromosomal copy number and massively parallel sequencing data from 947 human cancer cell lines, to facilitate the identification of genetic, lineage, and predictors of drug sensitivity.

\section{Membrane Immunostaining and Flow Cytometry}

Mononuclear bone marrow cells were obtained from AML patients and healthy donors with informed consent. The eligible patients AML patients (excludes acute promyelocytic leukemia) were all hematological relapsed (Bone marrow blasts $\geq 5 \%$; or reappearance of blasts in the blood) after chemotherapy or HSCT. 22 patients relapsed after HSCT, including 10 males and
12 females, with a median age of 29 (16-54) years old. 35 patients relapsed after chemotherapy, including 22 males and 13 females, with a median age of 41 (14-64) years old. Patients with complete remission and molecular relapse were excluded (Table 1).

Table 1. Clinic parameters of AML patients and healthy donors

\begin{tabular}{llll}
\hline Parameter & HSCT-R $(\mathrm{n}=22)$ & Chemo-R $(\mathrm{n}=35)$ & Donors $(\mathrm{n}=15)$ \\
\hline Age (years) & $29(16-54)$ & $41(14-64)$ & $36(6-64)$ \\
Sex & $10 \mathrm{M} ; 12 \mathrm{~F}$ & $22 \mathrm{M} ; 13 \mathrm{~F}$ & $8 \mathrm{M} ; 7 \mathrm{~F}$ \\
$\begin{array}{l}\text { Blast counts }(\%)( \pm \mathrm{SD}), \\
\mathrm{P}=0.20\end{array}$ & $53.8( \pm 30.4)$ & $41.5( \pm 28.8)$ & - \\
Mean Gal-9 counts $(\%)( \pm \mathrm{SD})$ & $31.4( \pm 43.3)$ & $13.5( \pm 26.1)$ & $0.9( \pm 0.64)$ \\
\hline
\end{tabular}

AML, acute myeloid leukemia; HSCT, hematopoietic stem cell transplantation; R, relapsed; Chemo, chemotherapy; M, male; F, female; Gal-9, Galectin-9; SD, standard deviation.

Single-cell suspensions of mononuclear bone marrow cells were stained with the anti-galectin-9 antibody (BD Biosciences) and analyzed by flow cytometry using the Cell Quest software (FACSCalibur; BD Biosciences).

\section{Co-expressed Genes of Gal-9}

The UALCAN database (http://ualcan.path.uab. edu/) was used to select the top 200 positively co-expressed genes of Gal-9, meanwhile the GEPIA dataset was applied to obtain top 200 co-expressed genes of Gal-9 for AML. The co-expressed genes obtained from the two databases were crossreferenced to obtain a cohort of 138 common co-expressed genes by a web tool (http://bioinformatics. psb.ugent.be/webtools/Venn/).

\section{Functional and KEGG Pathway Enrichment Analysis}

WebGestalt (WEB-based Gene SeT AnaLysis Toolkit) (http://www.webgestalt.org/) is a functional enrichment analysis web tool that reveals the biological meaning behind by entering a list of genes. Based on the candidate genes, gene ontology (GO) annotations can be divided into three categories: biological processes (BP), cellular components (CC) and molecular functions (MF) [14]. The KEGG pathway database is used to identify biological pathways for co-expressed genes enrichment [15]. We chose the top 10 terms to be the key pathways. False discovery rate $(F D R)<0.05$ was set as the cut-off criterion.

\section{Protein-Protein Interaction Network Construction and Hub Genes Identification}

Protein-Protein Interaction analysis (PPI) for co-expressed genes were performed by STRING database (https://string-db.org/) with an interaction score of $>0.4[16,17]$. Then, the Cytoscape software (version 3.8.0) was employed for visualizing 
molecular interaction networks. Each node is a gene, protein, or molecule, and the connections between nodes represent the interaction of these biological molecules, which can be used to identify interactions and pathway relationships between the proteins encoded by co-expressed gens in AML. The plug-in cytoHubba of Cytoscape was used to rank the genes by MCC method and the top 10 genes were determined as hub genes for further analysis.

\section{Gene Correlation and Survival Analysis in GEPIA}

The real hub genes and Gal-9 were performed gene expression correlation analysis by using given sets of TCGA expression data in GEPIA. The correlation coefficient was determined by the Pearson method. Gal-9 was presented on the x-axis, and hub genes were represented on the y-axis for tumor vs normal tissue analysis. Moreover, we used the GEPIA database to perform survival analysis for Gal-9 and the hub genes.

\section{Statistical Analysis}

Data in GEPIA databases, Oncomine databases and flow cytometry were analyzed by the student's t-test to compare the differential expression levels of Gal-9 mRNA or protein between two groups. Log-rank test was used for computing $P$-value in Kaplan-Meier univariate survival analyses. Pearson's correlation test was employed to evaluate the correlation between GAL-9 expression and hub gens. Fisher's exact test were performed to measure the gene enrichment in annotation terms and KEGG pathway. $P<0.05$ was considered as statistically significant difference.

\section{Results}

\section{Up-regulation of Gal-9 Expression in AML and High Gal-9 Expression}

\section{Predicts Poor Prognosis}

We analyzed the expression profile of Gal-9 using both Oncomine and GEPIA database. The expression of Gal-9 was significantly higher in the AML group than that in the normal group (all $P<0.05$, Figure 1A-D). Gal-9 has several isoforms, the mRNA expression level of LGALS9-005 ranked the highest among different protein coding types (Figure 1F). For validation, we performed meta-analysis of Gal-9 expression in 4 analyses with threshold by p-Value $\leq$ 0.05 , fold change $\geq 2$ and top $10 \%$ gene rank in Oncomine database. As shown in Figure 1B, compared with that in normal bone marrows, Gal-9 was significantly upregulated in AML $(P<0.05$, Figure 1B). In addition, CCLE analysis was consistent with that of ONCOMINE demonstrating that Gal-9 were distinctively up-regulated in AML cell lines

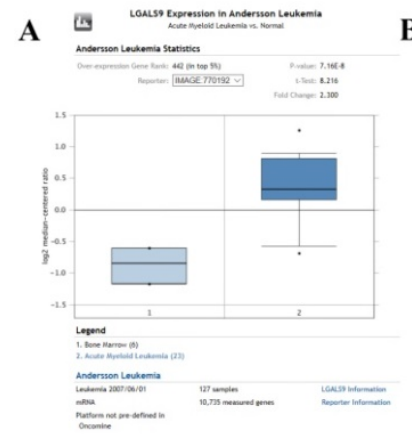

C

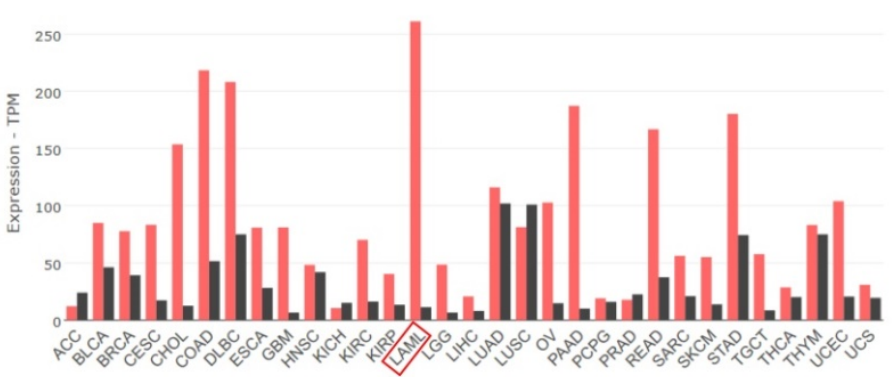

B

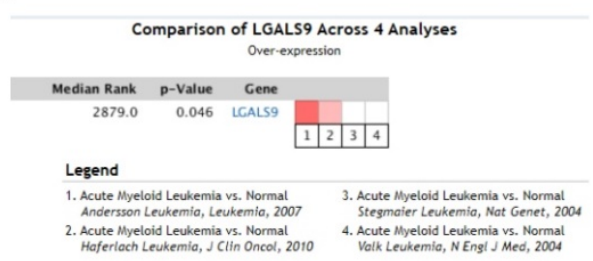

प Noc measured

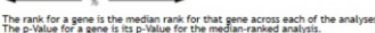

$10^{5} 2^{25} 251051$
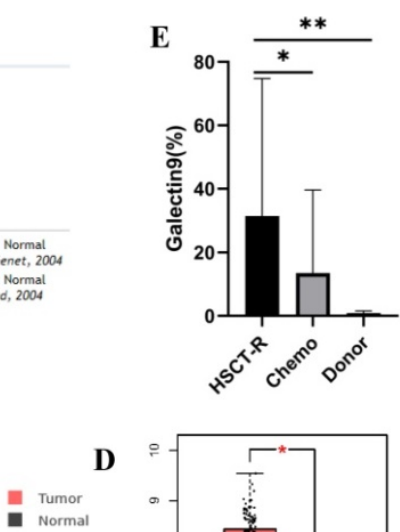

D

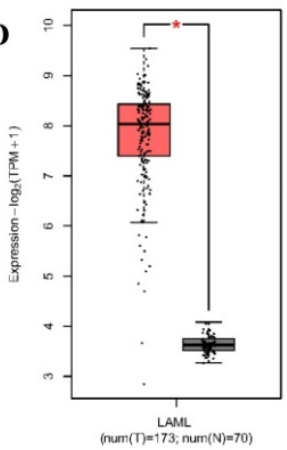

$\mathbf{F}$

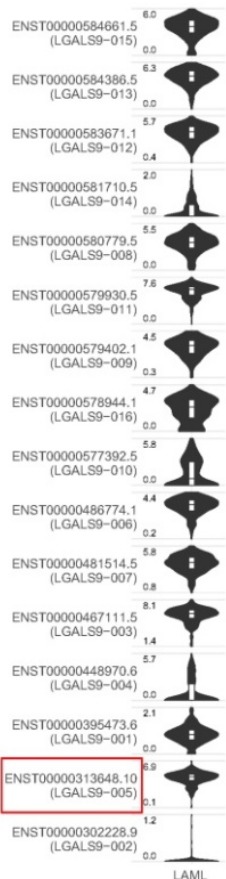

Figure 1. LGALS9 (Galectin-9) mRNA and protein expression was elevated in AML. (A) Box plots derived from gene expression data in ONCOMINE comparing expression of LGALS9 in normal and AML samples. The $p$ value was set up at 0.01 and fold change was defined as 2. (B) Meta-analysis of LGALS9 mRNA expression levels across 4 analyses in Oncomine database. (C,D) The GEPIA database verified that LGALS9 gene expression was significantly upregulated in AML samples (LAML) ( $n=173)$ compared with normal samples $(n=70), * P<0.05$. (E) Galectin-9 protein was highly expressed on the cell surface of AML cells, especially when relapse after HSCT compared with healthy donor derived mononuclear bone marrow cells and AML cells without HSCT in clinical practice. (F) LGALS9 has several isoforms, the mRNA expression level of LGALS9-005 ranked the highest among different protein coding types. 
(Figure 2A). In addition, Gal-9 protein was highly expressed on the cell surface of AML cells, especially when relapse after HSCT compared with healthy donor derived mononuclear bone marrow cells and AML cells without HSCT in clinical practice (Figure 1E; Table 1). These results implied that Gal-9 might play a unique role in the development of AML. Moreover, we used the GEPIA database to perform survival analysis for Gal-9 and found that increased expression of Gal-9 mRNA was significantly associated with poor overall survival (OS) in AML (Figure 2B).

\section{Functional and KEGG Pathway Enrichment Analysis Revealing Functional Association of Gal-9 with Immunoproteasome}

The UALCAN database was used to select the top 200 co-expressed genes of Gal-9. Meanwhile the GEPIA dataset was applied to obtain top 200 co-expressed genes of Gal-9 for AML. The co-expressed genes obtained from the two databases were cross referenced to obtain a cohort of 138 common co-expressed genes (Figure 3A). To analyze the biological classification of co-expressed genes, we used WebGestalt web tool for functional and pathway
A

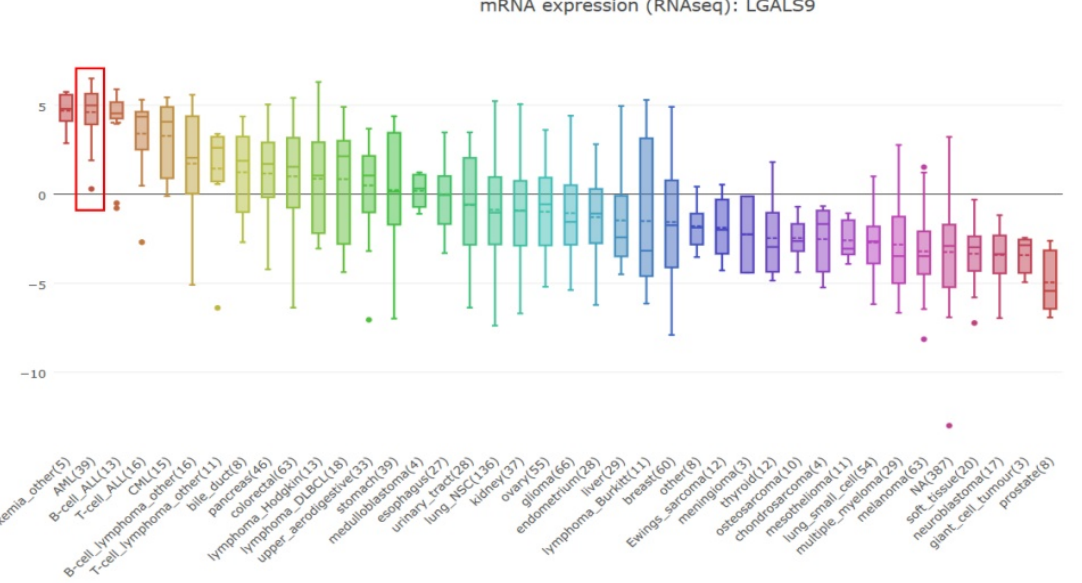

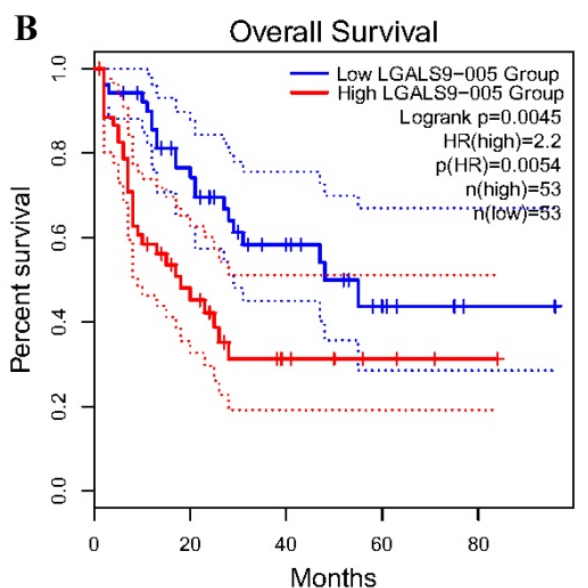

Figure 2. LGALS9 was distinctively high expressed AML cell lines from CCLE analysis and prognostic significances of LGALS9 gene expression. (A) The mRNA expression level of LGALS9 ranked the second highest in a variety of cancer cell line (shown in red frame). (B) LGALS9 overexpression predicts unfavorable overall survival in patients with AML.

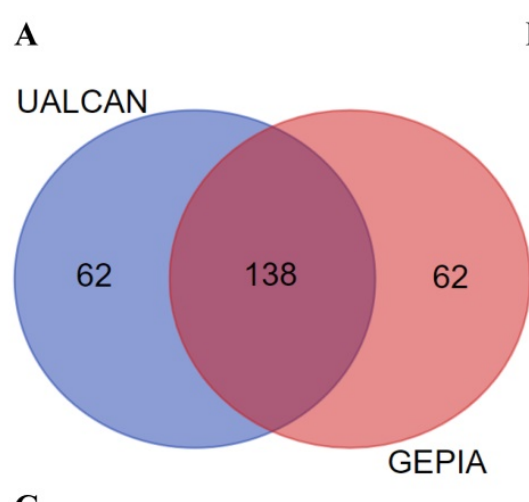

C

$=\mathrm{FDR} \leq 0.05 \quad \mathrm{FDR}>0.05$
B Bar chart of Biological Process categories

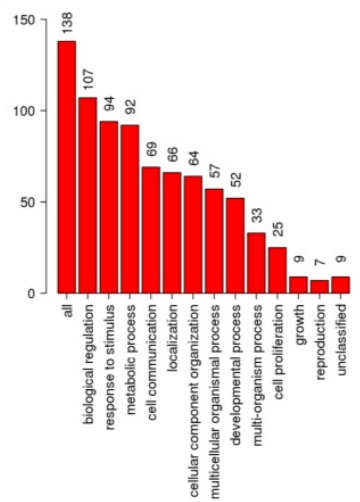

Bar chart of Cellular Component categories

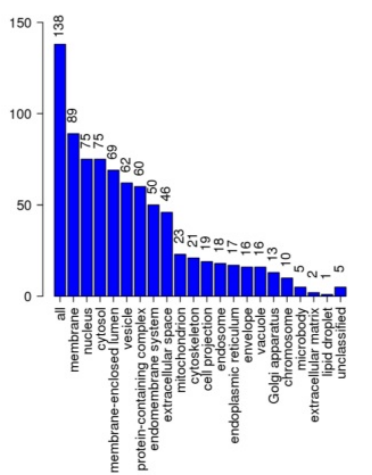

Bar chart of Molecular Function categories

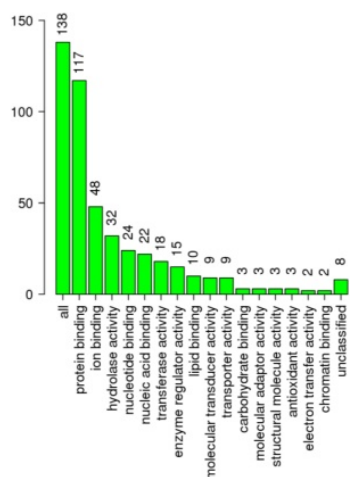

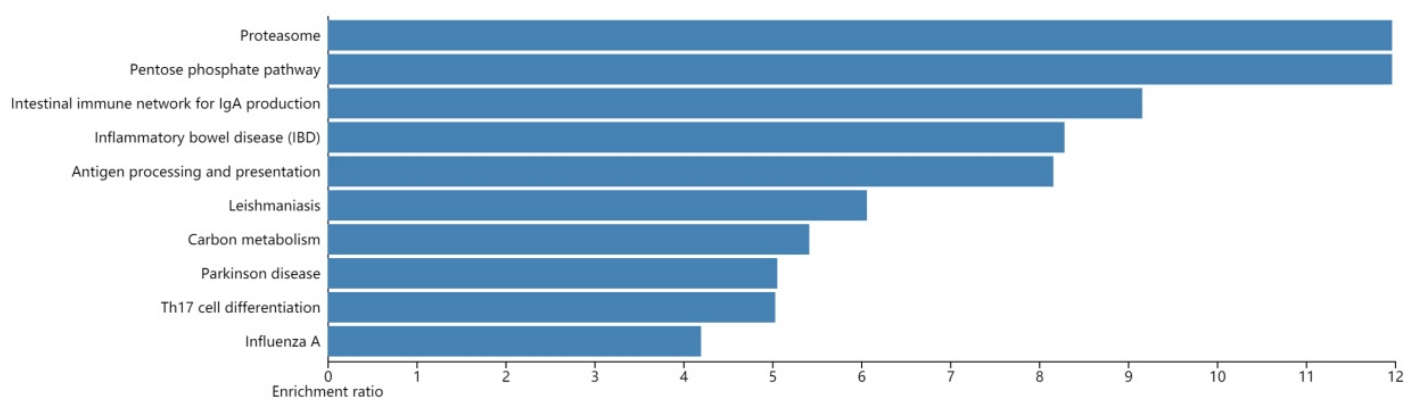

Figure 3. KEGG and GO enrichment analysis revealing functional association of LGALS9 with immunoproteasome. (A) Venn diagram represents the intersection of the top 200 positively corrected genes between the UALCAN database and the GEPIA database, 138 common co-expressed genes were obtained. (B) GO enrichment of co-expressed genes in biological process, cellular component and molecular function. (C) KEGG enrichment analysis of co-expressed genes with LGALS9. False discovery rate (FDR) < 0.05 was set as the cut-off criterion. 
enrichment analysis. GO analysis indicated that the biological processes including biological regulation, response to stimulus, metabolic process, cell communication, localization, cellular component organization, multicellular organismal process, developmental process, multi-organism process, cell proliferation were significantly affected, consistent with enrichment in respective cellular locations and proposed molecular functions (Figure 3B). KEGG pathway enrichment of Gal-9 interactive genes showed that proteasome, antigen processing and presentation, inflammatory bowel disease, Parkinson disease, intestinal immune network for $\operatorname{IgA}$ production, carbon metabolism, pentose phosphate pathway, influenza A, Th17 cell differentiation, Leishmaniasis were the most enriched pathways (Figure 3C). Collectively, these data suggest an essential role of Gal-9 in regulating many different biological functions such as inflammation, cell adhesion, cell proliferation, and cell apoptosis in AML. Interestingly, the function of Gal-9 in AML may be closely related to immunoproteasome.

\section{Gal-9 PPI Network Construction and Analysis of 10 Hub Genes}

Using the STRING database, the co-expressed 138 genes were constructed into a protein-protein network (Figure 4C). The top ten genes showing significant interaction including the PSMB8, PSMB10, PSME2, PSME1, IFI35, UBE2L6, PSMB4, ACO2, $\mathrm{MDH} 2$, ATP5B were identified as potential hub genes according to the MCC score generated by CytoHubba plug-in (Figure 4B). The KEGG pathway analysis of hub genes were further performed using WebGestalt web tool. Particularly, proteasomal ubiquitinindependent protein catabolic process and protein deubiquitination were the most enriched pathways, suggesting that they may participate in the protein anabolism required for cell survival and cell signaling (Figure 4D). Furthermore, the overall survival of hub genes was analyzed using Kaplan-Meier curve through the GEPIA database. 7 of these 10 hub genes exhibited poorer overall survival rate in higher expression groups (Figure 5). Amongst these hub genes, PSMB8 may be the most attractive target in AML. PSMB8 was highly expressed in AML and with poor prognosis (Figure 5). What's more, GEPIA correlation analysis showed that Gal-9 and PSMB8 had high correlation coefficients (Pearson's correlation $=0.62$ ) (Figure 4A). These data suggest that Gal-9 and immunoproteasome may play a cooperative role in the development of AML.

\section{Discussion}

AML is one of the most common lethal hematologic malignancies [1]. Despite wellcharacterized molecular and genetic characteristics, targeted therapies for AML have yet to lead to significantly improved clinical outcomes [1]. The pathogenesis, diagnosis and treatment of AML remains the matter of intense research currently.

This study was the first to show the mRNA expression and prognosis of Gal-9 in AML, although other studies have reported the role of Gal-9 in tumorigenesis, development, metastasis and potential therapy of several cancers $[7,8]$. As a member of the galectin family, more and more research revealed that Gal-9 is a negative regulator of tumor immune responses by promoting Th1, Th17 and cytotoxic T cells exhaustion, driving the expansion of myeloid-derived suppressor cells and FoxP3+Tregs when interactions with its ligand Tim-3 [8, 10, 11]. Gal-9 plays an essential role in regulating inflammation, cell adhesion, cell proliferation, and cell apoptosis $[8,10,11]$. Our study has revealed that Gal-9 was overexpressed in AML, what's more, Gal-9 protein was highly expressed on the cell surface of AML cells, especially when relapse after transplant compared with healthy donor derived mononuclear bone marrow cells and AML cells without HSCT in clinical practice. The Gal-9 associated co-expressed genes are primarily involved in immune response and antigen processing and presentation, these data suggest that Gal-9 may play an important role in immune escape of AML and consistent with the indication of poor prognosis in AML patients. Thus, targeting Gal-9 may be a promising strategy for therapeutic intervention against AML. So far, some strategies appear to be promising in abrogating galectin-mediated effects. These include: (1) neutralization of galectin-binding through blocking antibodies against galectins [18], (2) competitive inhibition of galectin binding via synthetic analogs of galectin carbohydrate-binding determinants [19], (3) modification of the glycosylation profiles necessary for galectin binding on anti-tumor immunocytes through fluorinated analogs of glucosamine [7, 20]. Recently, a Phase IB (NCT03066648) clinical trial was undertaken to investigate the therapeutic effects on blocking galectin-9/TIM-3 pathway with anti-TIM-3 antibody, about one-third newly diagnosed and relapse/refractory AML patients achieved complete or partial response [21]. Indeed, treatment with anti-Tim-3 alone has little or no effect because of the complex and multiple biological features of AML. Combined treatment is still the future direction for AML. 
A

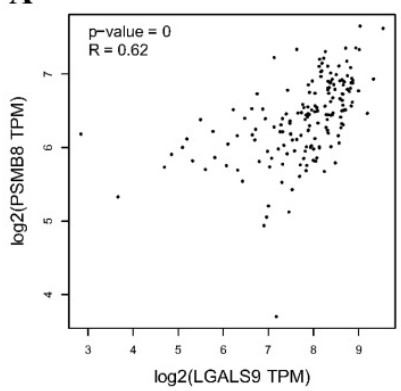

B

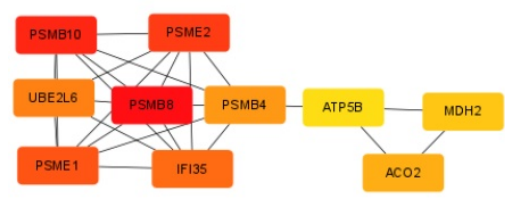

C

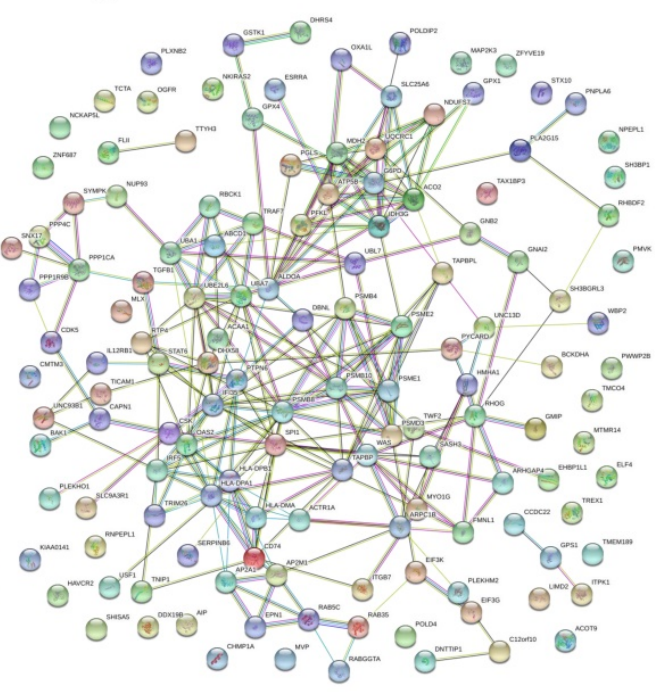

D

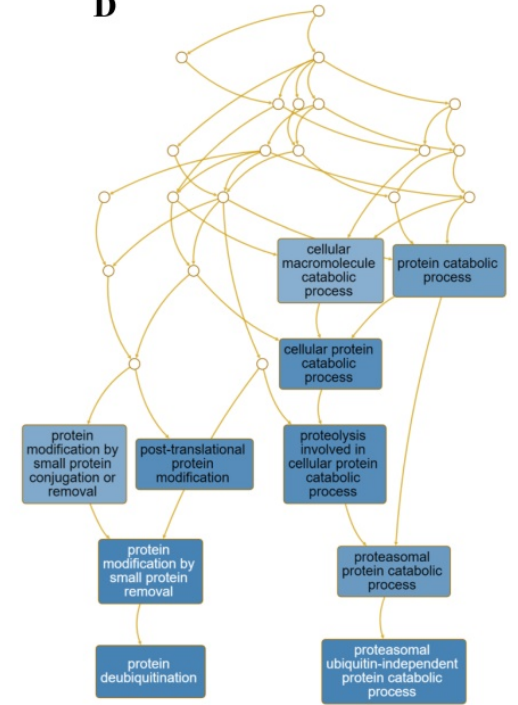

Figure 4. Construction of PPI network of LGALS9 positive-correlation genes and analysis of hub genes. (A) Correlation between LGALS9 and PSMB8 mRNA expression determined using GEPIA database. (B) The top ten hub genes were identified using cytoHubba tool kits in Cytoscape. (C) Clustering analysis of LGALS9 co-expressed genes by STRING tools. (D) The KEGG pathway analysis of hub genes using WebGestalt web tool.
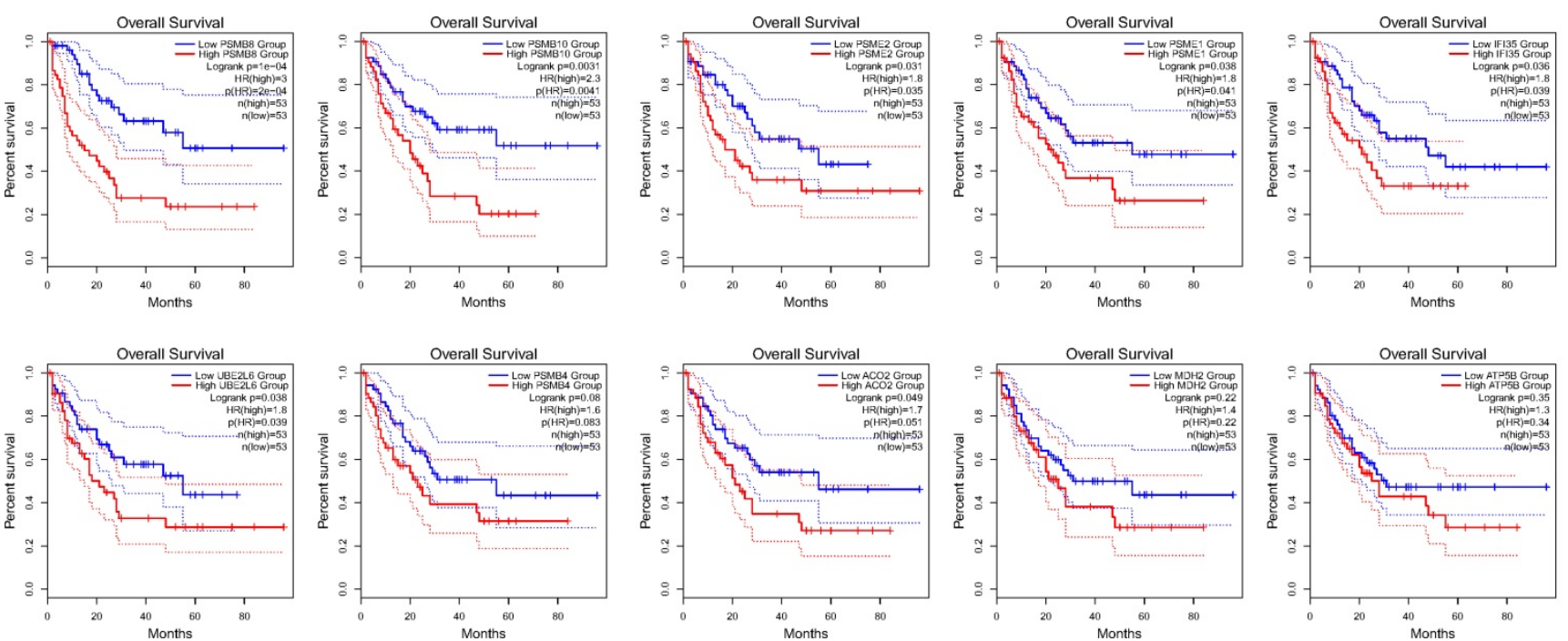

Figure 5. Over survival analyses of hub genes in AML using GEPIA database. 7 of the 10 hub genes exhibited poorer overall survival rate in higher expression groups.

PSMB8 is a member of the proteasome $\beta$-type family, which is also named the large multifunctional protease 7 (LMP7), encodes $\beta 5$ i which often functions as a subunit of the immunoproteasome [22]. PSMB8 encodes $\beta 5 \mathrm{i}$ which performs as one of the catalytic subunits of immunoproteasome and takes part in immunological reaction via 265 proteasome mediated degradation [22]. PSMB8 plays an important role in promoting cell survival and progression of the leukemia through PSMB8-mediated PI3K/AKT pathway activation [23] and constitutive nuclear factor $\kappa B \quad(N F-\kappa B)$ signaling, while NF- $\mathrm{B}$ is constitutively active in leukemic stem cells (LSCs), but not in normal hematopoietic progenitor cells [24-26]. The expression of PSMB8 was remarkably correlated with Ki-67, which is always considered as a cancer marker [27]. These findings indicate that PSMB8 may be used as a novel prognostic factor and therapeutic target of AML.

In this study, we found PSMB8 was highly expressed in AML with poor prognosis. The positive correlation of Gal-9 and PSMB8 in AML may indicate a synergy in cell survival, cell signaling and the development of AML, co-targeting these two genes might be an efficient way to treat AML.

For most patients with AML, allogeneic hematopoietic cell transplantation is the only curative treatment, however relapse is the common cause of death and its incidence and outcome have not significantly improved over the last decades [28]. The relapse of AML after HSCT is associated with immune escape. The mechanisms of immune evasion 
are complex and diverse, mainly including abrogation of leukemia cell recognition due to loss of HLA genes, immunosuppression by immune-checkpoint ligand expression, production of anti-inflammatory factors, release of metabolically active enzymes, loss of proinflammatory cytokine production, and acquisition of novel driver mutations that promote leukemia outgrowth [29, 30]. In our study, Gal-9 protein was highly expressed on the cell surface of AML cells, especially when relapse after transplant, indicating its important role in immune escape.

In summary, we have confirmed the overexpression of Gal-9 and its partner PSMB8 in AML and validated their importance as prognostic factors. We propose that Gal-9 and PSMB8 could be a promising molecular target for treatment of AML and may provide more combined treatment options, especially in patients with relapse after HSCT.

\section{Acknowledgements}

This work was supported by the Young Scholars of Aerospace Center Hospital (Grant No.2018QN05) and the China Capital Characteristic Clinic Project (Grant No. Z171100001017103).

\section{Competing Interests}

The authors have declared that no competing interest exists.

\section{References}

1. Döhner H, Weisdorf DJ, Bloomfield CD. Acute Myeloid Leukemia. The New England journal of medicine. 2015; 373: 1136-52.

2. Kishtagari A, Levine RL, Viny AD. Driver mutations in acute myeloid leukemia. Current opinion in hematology. 2020; 27: 49-57.

3. Vosberg S, Greif PA. Clonal evolution of acute myeloid leukemia from diagnosis to relapse. Genes, chromosomes \& cancer. 2019; 58: 839-49.

4. Lai C, Doucette K, Norsworthy K. Recent drug approvals for acute myeloid leukemia. Journal of hematology \& oncology. 2019; 12: 100.

5. Blum WG, Mims AS. Treating acute myeloid leukemia in the modern era: A primer. Cancer. 2020; 0:1-10.

6. Matsumoto R, Hirashima M, Kita H, Gleich GJ. Biological activities of ecalectin: a novel eosinophil-activating factor. Journal of immunology (Baltimore, Md : 1950). 2002; 168: 1961-7.

7. Fujihara S, Mori H, Kobara H, Rafiq K, Niki T, Hirashima M, et al. Galectin-9 in cancer therapy. Recent patents on endocrine, metabolic \& immune drug discovery. 2013; 7: 130-7.

8. Fujita K, Iwama H, Oura K, Tadokoro T, Samukawa E, Sakamoto T, et al Cancer Therapy Due to Apoptosis: Galectin-9. International journal of molecular sciences. 2017; 18:84.

9. Rabinovich GA, Rubinstein N, Toscano MA. Role of galectins in inflammatory and immunomodulatory processes. Biochimica et biophysica acta. 2002; 1572: 274-84.

10. Zhou Q, Munger ME, Veenstra RG, Weigel BJ, Hirashima M, Munn DH, et al. Coexpression of Tim-3 and PD-1 identifies a CD8+ T-cell exhaustion phenotype in mice with disseminated acute myelogenous leukemia. Blood. 2011; 117: 4501-10.

11. Cedeno-Laurent F, Dimitroff CJ. Galectins and their ligands: negative regulators of anti-tumor immunity. Glycoconjugate journal. 2012; 29: 619-25.

12. Seki M, Oomizu S, Sakata KM, Sakata A, Arikawa T, Watanabe K, et al. Galectin-9 suppresses the generation of Th17, promotes the induction of regulatory $\mathrm{T}$ cells, and regulates experimental autoimmune arthritis. Clinical immunology (Orlando, Fla). 2008; 127: 78-88.

13. Tang Z, Li C, Kang B, Gao G, Li C, Zhang Z. GEPIA: a web server for cancer and normal gene expression profiling and interactive analyses. Nucleic acids research. 2017; 45: W98-w102.

14. The Gene Ontology (GO) project in 2006. Nucleic acids research. 2006; 34: D322-6
15. Kanehisa M, Goto S. KEGG: kyoto encyclopedia of genes and genomes. Nucleic acids research. 2000; 28: 27-30

16. Szklarczyk D, Franceschini A, Kuhn M, Simonovic M, Roth A, Minguez P, et al. The STRING database in 2011: functional interaction networks of proteins, globally integrated and scored. Nucleic acids research. 2011; 39: D561-8.

17. Franceschini A, Szklarczyk D, Frankild S, Kuhn M, Simonovic M, Roth A, et al. STRING v9.1: protein-protein interaction networks, with increased coverage and integration. Nucleic acids research. 2013; 41: D808-15.

18. Klibi J, Niki T, Riedel A, Pioche-Durieu C, Souquere S, Rubinstein E, et al. Blood diffusion and Th1-suppressive effects of galectin-9-containing exosomes released by Epstein-Barr virus-infected nasopharyngeal carcinoma cells. Blood. 2009; 113: 1957-66

19. Rabinovich GA, Cumashi A, Bianco GA, Ciavardelli D, Iurisci I, D'Egidio M, et al. Synthetic lactulose amines: novel class of anticancer agents that induce tumor-cell apoptosis and inhibit galectin-mediated homotypic cell aggregation and endothelial cell morphogenesis. Glycobiology. 2006; 16: 210-20.

20. Woynarowska B, Skrincosky DM, Haag A, Sharma M, Matta K, Bernacki RJ. Inhibition of lectin-mediated ovarian tumor cell adhesion by sugar analogs. The Journal of biological chemistry. 1994; 269: 22797-803.

21. Borate U, Esteve J, Porkka K, Knapper S, Vey N, Scholl S, et al. Phase Ib Study of the Anti-TIM-3 Antibody MBG453 in Combination with Decitabine in Patients with High-Risk Myelodysplastic Syndrome (MDS) and Acute Myeloid Leukemia (AML). Blood. 2019; 134: 570-

22. Rivett AJ, Hearn AR. Proteasome function in antigen presentation: immunoproteasome complexes, Peptide production, and interactions with viral proteins. Current protein \& peptide science. 2004; 5: 153-61.

23. Lei $M$, Jingiing $Z$, Tao J, Jianping $M$, Yuanxin $Z$, Jifeng $W$, et al. LncRNA HCP5 promotes LAML progression via PSMB8-mediated PI3K/AKT pathway activation. Naunyn-Schmiedeberg's archives of pharmacology. 2020; 393: 1025-32.

24. Guzman ML, Neering SJ, Upchurch D, Grimes B, Howard DS, Rizzieri DA, et al. Nuclear factor-kappaB is constitutively activated in primitive human acute myelogenous leukemia cells. Blood. 2001; 98: 2301-7.

25. Kagoya Y, Yoshimi A, Kataoka K, Nakagawa M, Kumano K, Arai S, et al. Positive feedback between NF-kB and TNF-a promotes leukemia-initiating cell capacity. The Journal of clinical investigation. 2014; 124 : 528-42.

26. Csizmar CM, Kim DH, Sachs Z. The role of the proteasome in AML. Blood cancer journal. 2016; 6: e503.

27. Su C, Liu C, Zhao L, Jiang J, Zhang J, Li S, et al. Amide Proton Transfer Imaging Allows Detection of Glioma Grades and Tumor Proliferation: Comparison with Ki-67 Expression and Proton MR Spectroscopy Imaging. AJNR American journal of neuroradiology. 2017; 38: 1702-9.

28. Horowitz M, Schreiber H, Elder A, Heidenreich O, Vormoor J, Toffalori C, et al. Epidemiology and biology of relapse after stem cell transplantation. Bone marrow transplantation. 2018; 53: 1379-89.

29. Christopher MJ, Petti AA, Rettig MP, Miller CA, Chendamarai E, Duncavage EJ, et al. Immune Escape of Relapsed AML Cells after Allogeneic Transplantation. The New England journal of medicine. 2018; 379: 2330-41.

30. Zeiser R, Vago L. Mechanisms of immune escape after allogeneic hematopoietic cell transplantation. Blood. 2019; 133: 1290-7. 